\title{
DIFFUSE IONIZED GAS IN NEARBY GALAXIES
}

\author{
R.A.M. WALTERBOS \\ Astronomy Department, University of California \\ Berkeley, CA 94720, USA
}

\begin{abstract}
We discuss the distribution and spectral characteristics of diffuse ionized gas in nearby galaxies. The existence of this elusive component of the interstellar medium (ISM), also referred to as the Warm Ionized Medium, is by now well established from deep imaging and spectroscopic surveys in several emission lines in external galaxies. Diffuse ionized gas is characterized by a relatively high ratio of [SII] over $\mathrm{H} \alpha$ intensities, typically twice as high as for discrete HII regions. The diffuse gas has been mapped in both edge-on and more face-on galaxies providing information on the radial and vertical distribitions. Emission from diffuse ionized gas is strongest around star forming regions. The vertical distribution appears related to the radio continuum thick-disk emission. We also briefly discuss ionization mechanisms, and the connection between star formation characteristics and morphology of the interstellar medium.
\end{abstract}

\section{INTRODUCTION}

The existence of diffuse ionized gas in the interstellar medium of the Galaxy has been established in several independent ways. Evidence for this widely distributed, low-density $\left(n_{e} \sim 0.2 \mathrm{~cm}^{-3}\right)$, relatively cool $(<10,000 \mathrm{~K})$ ionized gas follows from imagery and spectroscopy in emission lines, especially the $\mathrm{H} \alpha$ line, from pulsar dispersion measurements, observations of scintillation of compact radio sources, absorption of non-thermal radio emission, Faraday rotation, and thermal radio continuum emission. The first two probes have provided most information on the distribution and characterisctics of this gas in the general solar neighborhood (see Reynolds, this volume). The gas is referred to as Diffuse Ionized Gas (DIG) or Warm Ionized Medium (WIM). We will use the latter acronym in this paper. With its large vertical extent, more than a kpc above the disk in our Galaxy, and significant filling factor, $\geq 0.2$, the WIM evidently plays an important role in the disk-halo interface.

There are several reasons why it is necessary to study this component of the ISM in galaxies other than our own. First, the global distribution of the WIM in our Galaxy is not known because of our location in the disk. Second, the connection 
between the young stellar population, presumably responsible for ionizing the gas, and the WIM is difficult to derive for the same reason. Thirdly, if OB stars power the ISM, then ISM properties are likely to depend on galaxy type and on position in the disk, since it is well established that star formation characteristics vary strongly both with galaxy type and galactic radius (e.g. Kennicutt, 1983, 1989). Hence it is important to study the WIM in a variety of galaxies. By observing both edge-on and more face-on systems, all these issues can be addressed.

\section{OBSERVATIONS}

Of the above-mentioned techniques that are in principle able to detect the WIM in external galaxies, direct imagery and spectroscopy of (optical) emission lines are most promising with current observational capabilities. The thermal radio continuum emission of the WIM is very weak and difficult to separate from the generally much stronger non-thermal synchrotron emission and from the contribution of discrete HII regions, especially at the low resolution of most radio surveys. To detect the turnover in the non-thermal radio spectrum due to absorption by thermal electrons requires observations at very low frequencies at which not many radio telescopes operate (see Israel, this volume). Finally, with the exception of the Magellanic Clouds, galaxies are too far away to detect radio pulsars. Thus we are left with optical imagery and spectroscopy.

The study of the morphology of the WIM in nearby galaxies is not new, of course. Photographic surveys of the Magellanic Clouds (Davies et al., 1976, Meaburn, 1980), M31 (Pellet et al., 1978), M33 (e.g. Courtès et al., 1988), and other galaxies (e.g. Monnet, 1971, Sabbadin and Bianchi, 1979, Hunter, 1982, Hodge and Kennicutt, 1983) have shown filaments, shells, and bubbles of ionized gas, up to more than a kpc in diameter. Some of these surveys also pointed out the existence of low-intensity diffuse ionized gas. Strictly speaking, filaments or bubbles are not diffuse gas, in the sense of having a homogeneous distribution. One of the characteristic optical signatures of the WIM is a relatively high ratio of forbidden sulfur, [SII], over $\mathrm{H} \alpha$ line intensities, typically twice as high as discrete HII regions, but not as high as is found in supernova remnants. If we adopt that characteristic of the WIM as a diagnostic, the faint loops and shells can actually be seen as part of the WIM, since they share this property. This high line ratio is characteristic of gas ionized by a strongly diluted photon field (or shocks) and as such both diffuse gas and loops may be part of the same phenomenon. Lasker $(1977,1979)$ obtained $\mathrm{H} \alpha$ and [SII] photographs of several loops in the LMC and drew attention to their relatively high ratio of $[\mathrm{SII}] / \mathrm{H} \alpha$ emission.

The earlier photographic surveys typically reached emission measures, $E M=$ $\int n_{e}^{2} d l$, where $n_{e}$ is the electron density and the integral is along the line of sight, down to $40 \mathrm{pc} \mathrm{cm}^{-6}$, and suffered from variations in the plate background, which limited quantitative work. Observations of the WIM in the Galaxy by Reynolds $(1987,1988)$ show emission measures down to a few $\mathrm{pc} \mathrm{cm}^{-6}$, well below the photographic limit. However, such faint limits can be reached with CCD detectors 
on even rather modest telescopes. CCD observations of the WIM in other galaxies have only been obtained over the past few years, and allow the first detailed studies of this component of the ISM.

\section{DISTRIBUTION AND SPECTRAL CHARACTERISTICS}

\subsection{The WIM in Nearby Spiral and Irregular Galaxies}

From a study of the $\mathrm{H} \alpha$ luminosity functions in several spiral galaxies, Kennicutt et al. (1989) concluded that a substantial fraction of the total $\mathrm{H} \alpha$ luminosity from a galaxy is contributed by diffuse ionized gas, rather than discrete HII regions. For example, for the Large Magellanic Cloud, at least 20-30\% of the total $\mathrm{H} \alpha$ emission comes from diffuse gas (Kennicutt and Hodge, 1986). Diffuse gas shows up clearly in two recent very deep CCD surveys of M33 (Hester and Kulkarni, 1990) and M31 (Walterbos and Braun, 1990a, 1990b), and it has also been found in several edge-on systems, which will be discussed below. Fig. 1 shows a field in the NE arm of M31, which was obtained with the No-1 36" telescope at Kitt Peak. The WIM shows up both as faint shells and loops, and as diffuse gas with no apparent structure. That the gas has truely different characteristics from the normal, discreet HII regions is apparent from Fig. 2, which shows cross-cuts through a discrete HII region and through a region with mainly diffuse gas. The ratio of $[\mathrm{SII}] / \mathrm{H} \alpha$ intensities is consistently higher for the WIM than for the discreet HII regions, just as is found in our Galaxy (Reynolds, this volume). The diffuse gas can be traced to emission measures well below $5 \mathrm{pc} \mathrm{cm}^{-6}$.

Our survey consisted of 19 fields, imaged in both $\mathrm{H} \alpha$ and [SII], which cover large sections of the spiral arms in the Northern half of M31, over a range in radius from 4 to $15 \mathrm{kpc}$. The ratio of $[\mathrm{SII}] / \mathrm{H} \alpha$ intensities in the WIM is 0.5 , and does not seem to vary with radius. A systematic decrease in that ratio with distance from the center is observed for discreet HII regions. The emission from the WIM is strongest closest to the major star forming regions. This is apparent in Fig. 1 and is generally true across the galaxy.

Intensities for the gas with high $[\mathrm{SII}] / \mathrm{H} \alpha$ ratio reach up to $E M=80 \mathrm{pc} \mathrm{cm}^{-6}$, which is a few times higher than the maximum intensities that Reynolds (1985) finds for our Galaxy. However, this may not be surprising in view of the fact that our fields are located in bright spiral arms. Direct summation of the flux of the WIM indeed shows that it contributes between 20 to $40 \%$ of the total $\mathrm{H} \alpha$ flux in M31. The exact value depends on where the transition from WIM to discreet HII region is assumed to take place. Also, our frames do not cover the interarm regions, and intensities were assumed to be zero in the corners of the frames.

The survey of M33 by Hester and Kulkarni shows very similar results. No [SII] maps have been published yet, so it is not yet known if a similar change in ratio of [SII] $/ \mathrm{H} \alpha$ intensities occurs, but the presence of the WIM is evident from their high quality image. Their frame covers both arm and interarm regions. Weak interarm 


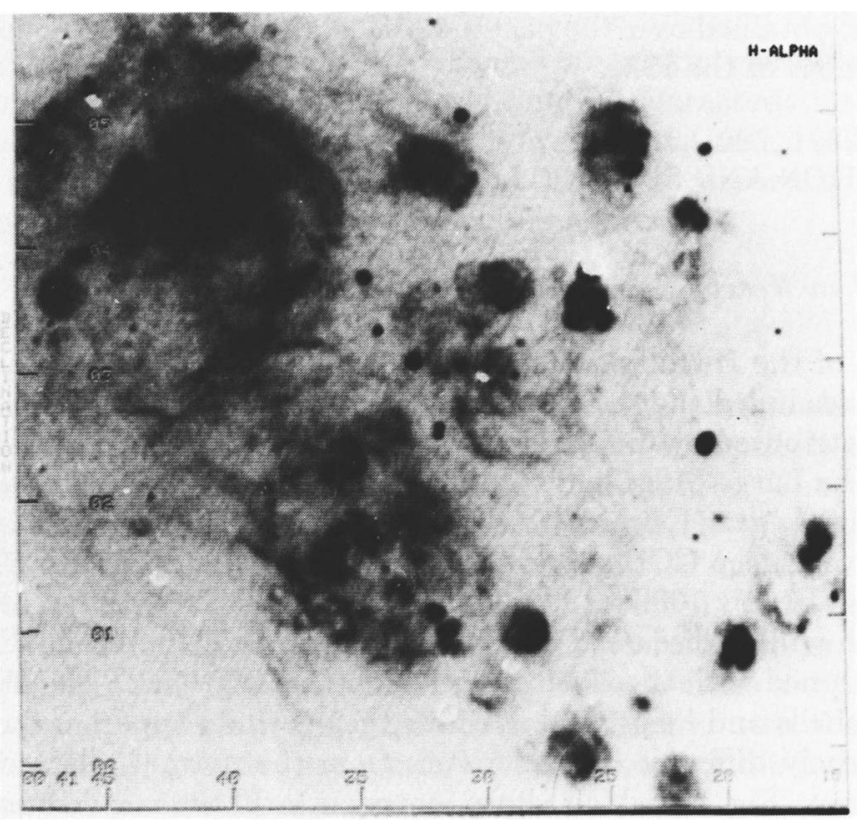

Figure 1. Continuum-subtracted CCD frame in $\mathrm{H} \alpha$ of a region in the NE arm at $10 \mathrm{kpc}$ distance from the center of M31. The frame measures 6.6 arcmin or $1.3 \mathrm{kpc}$ on the side. North is up. The bright HII region in the top-left corner is Pellet 550, one of the largest HII regions in M31. The WIM shows up as faint diffuse emission surrounding the discrete HII regions; also many of the faint loops and shells have spectral characteristics similar to that of the WIM (see text).

emission is seen, but the intensities are higher in the spiral arms. The average emission measure of the WIM is about $40 \mathrm{pc} \mathrm{cm}^{-6}$. Various loops and shells are seen, more homegeneous diffuse gas and possibly sheets of ionized gas (Hester and Kulkarni, 1990). The presence of the WIM in the interarm regions in M33 is not surprising, since there are massive stars there as well (Freedman, 1984).

The WIM has also been detected in irregular galaxies, other than the LMC. In particular, Hunter and Gallagher (1989) drew attention to various loops and filaments in their survey of several irregular galaxies, which have the characteristic line intensity ratios of the WIM. In this case, however, some of the loops were far displaced from $\mathrm{O}$ stars so the source of ionization may be a problem. Also, the emission measures are much higher, reaching values of several hundred $\mathrm{pc} \mathrm{cm}^{-6}$. Still, in view of the spectral characteristics, this material, which they refer to as froth, should be considered as part of the WIM. 

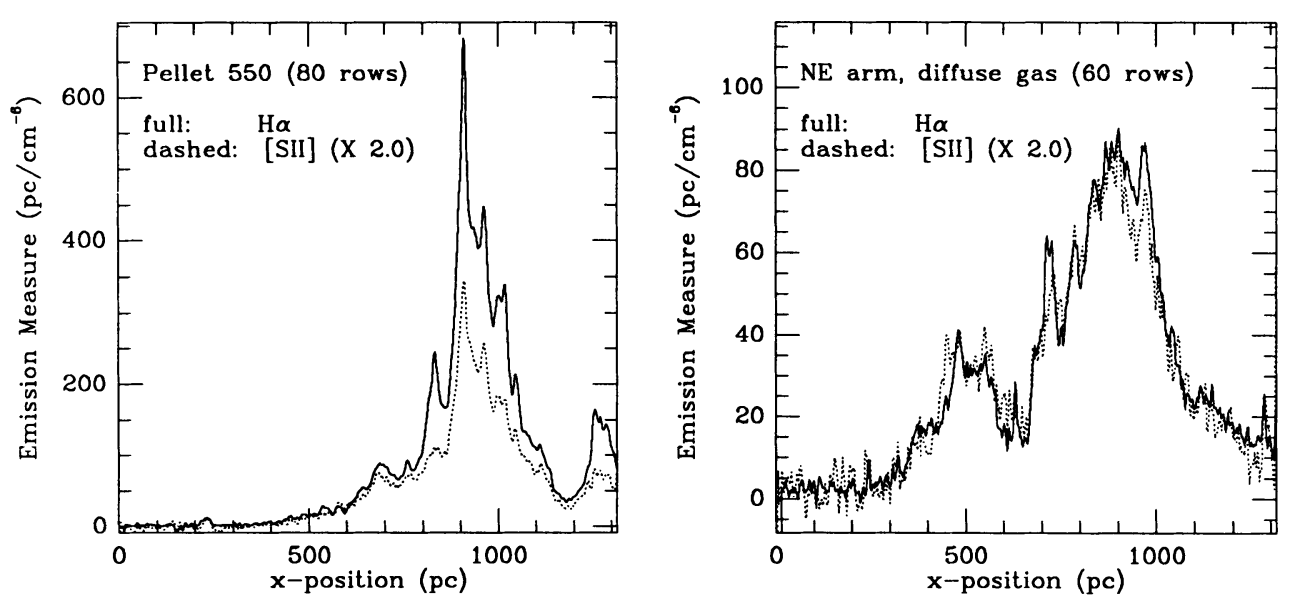

Figure 2. Two cross-cuts in $\mathrm{H} \alpha$ and [SII] emission through different regions in the NE spiral arm in M31. The [SII]-intensities have been multiplied by two. The cuts are averages of the indicated number of rows in a CCD frame. The left plot contains the bright HII region Pellet 550, while the right plot corresponds to a region with mainly diffuse gas.

\subsection{The Vertical Distribution}

Some edge-on galaxies have been mapped recently, to determine the distribution of the WIM above the disk. In the Milky Way, the WIM has a scaleheight of about $1 \mathrm{kpc}$, significantly larger than that of atomic hydrogen (see Reynolds, this volume). The best example to date of a galaxy with a prominent $\mathrm{H} \alpha$ disk is NGC 891, an $\mathrm{Sb}$ galaxy that is a close twin of our Galaxy in its optical structure (van der Kruit, 1984). Rand et al. (1990) and Dettmar (1990) found a very extended $\mathrm{H} \alpha$ disk, out to some $4 \mathrm{kpc}$ above the plane. This galaxy may also show evidence for outflow of material from the disk through chimneys (Norman and Ikeuchi, 1989), the walls of which show up as ionized filaments protruding from the disk. More details about these and other observations of NGC 891 can be found elsewhere in this volume. Hester et al. also present some results for NGC3079 in these proceedings.

A somewhat different case than NGC891 is presented by NGC4244, a nearby Sc edge-on system. We recently obtained $\mathrm{H} \alpha$ imagery with the No-1 36" telescope at Kitt Peak of this and other galaxies (Walterbos, Braun and Kennicutt, in prep.). Fig. 3 shows a grey scale plot of the continuum-subtracted image. No thick $\mathrm{H} \alpha$ disk is evident in our images. Our observational parameters were quite similar to those of Dettmar in his study of NGC891, so if a comparable disk were present, we ought to have seen it. The full width at $E M=10 \mathrm{pc} \mathrm{cm}^{-6}$ is about $800 \mathrm{pc}$, while it is $2000 \mathrm{pc}$ in NGC891 (Rand et al., 1990). Our current data do not rule out the presence of a weaker disk and deeper exposures would be useful. What is noteworthy is that the distribution of HII regions is remarkably wide in the $\mathrm{z}$ direction. HII regions are generally found out to $300 \mathrm{pc}$ above the plane of the 
disk. The inclination of this galaxy is 88 degrees so some of the apparent width results from projection effects, but this is not the case near the outer edges. One remarkable HII region is found at an apparent height of $750 \mathrm{pc}$ above the disk. Its luminosity is comparable to that of Orion in the Milky Way, so it is a small star forming complex. It might be an outlying HII region in the warped part of the disk. It will be interesting to compare the ionized gas distribution with high resolution HI data. We have recently observed this galaxy at the VLA (Braun et $a l$. , in prep.).

A trend appears which suggests that thick $\mathrm{H} \alpha$ disks occur in galaxies that have thick radio continuum disks. NGC891 is known to have a prominent nonthermal radio continuum disk (Allen, these proceedings), as was also pointed out in the papers by Rand et al. and Dettmar. Also our Galaxy has a thick radio disk (Beuermann et al., 1985). The extent of the radio disk is very similar to that of the $\mathrm{H} \alpha$ emission in both cases. NGC4244, on the other hand, has not been detected in radio continuum (Hummel et al., 1984). The underlying mechanism that may explain both the thick radio and $\mathrm{H} \alpha$ disks is most likely the level of star formation. Galaxies with low star formation activity do not manage to vent material into the halo and thus there are no channels for either the gas or the ionizing photons to reach high z-distances. To illustrate this, we can look at the rate of star formation per unit area for these systems. Although not all the far-infrared emission is directly related to star formation activity, it may be the best indicator for edge-on systems where optical tracers suffer too much from extinction. Using data from Rice et al. (1988), the average far-infrared luminisity per unit area, in solar luminosities per square pc, is 4.1 for NGC891 and 0.4 for NGC4244, which is even lower than a value of 0.8 for M31, a galaxy known to have very low star formation activity (Walterbos, 1988). Evidently this correlation can easily be tested by observations of more galaxies.

\section{SOURCES OF IONIZATION}

Originally, the relatively high ratios of $[\mathrm{SII}] / \mathrm{H} \alpha$ intensities that were observed for the WIM seemed to point to shocks as the most likely source of ionization, since spectra of supernova remnants, where shocks are clearly important, show high values for this ratio, between 0.5 and 1.2 (e.g. Blair et al., 1981). Models of shock ionization (e.g. Brand and Mathis, 1978) indeed produce high ratios, but the problem is that the observed values in the WIM in M31 and also in NGC891 are extremely constant, whereas the ratio is critically dependent on shock velocity. A problem is also that all the energy from supernovae would be required to ionize the WIM in the Galaxy (Reynolds, 1988). Photo ionization models generally do not reproduce the high ratios of $[\mathrm{SII}] / \mathrm{H} \alpha$ emission. However, Mathis (1986) calculated the effects of a very dilute photon field in a tenuous medium, as is the case for the WIM, and did reproduce the observed line ratios. Thus, the current data seem to be more in agreement with OB stars as the major ionizing source for the WIM. Also energetically, this seems to be feasible (Reynolds, 1988). There are 


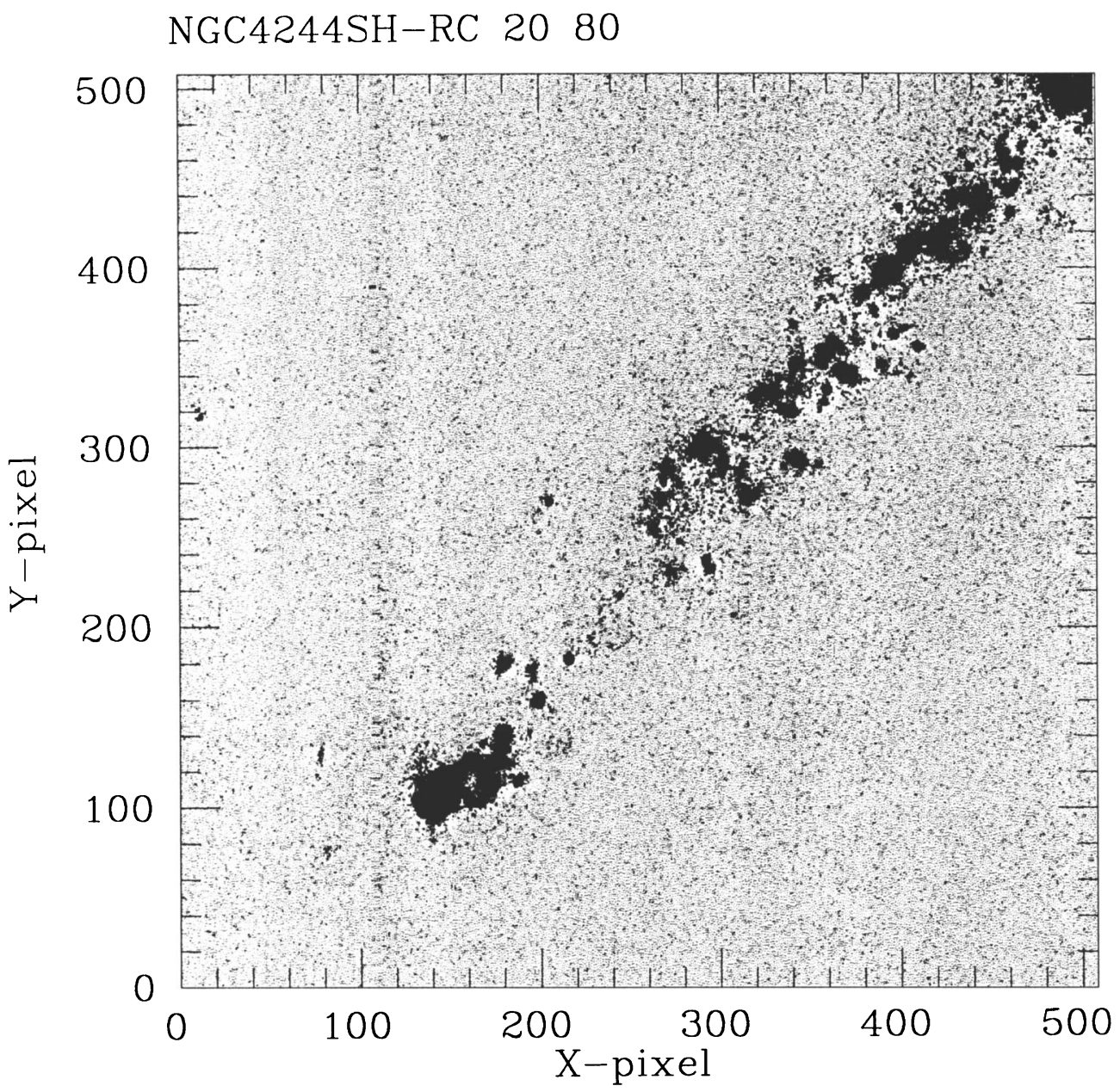

Figure 3. $\mathrm{H} \alpha$-image of the SW part of the edge-on galaxy NGC4244, obtained with the No1 36" telescope at Kitt Peak National Observatory. Scale: 100 pixels equals about $1100 \mathrm{pc}$. Note the wide distribution of HII regions; the one at $X=$ $205, Y=270$ is about $750 \mathrm{pc}$ above the plane. This galaxy does not seem to have a prominent thick $\mathrm{H} \alpha$ disk, however.

two potential problems with the photo ionization model though. First, it is not clear how the ionizing photons can reach such large distances above the plane; this may put strong restrictions on the morphology of the interstellar medium (see also Norman, these proceedings). Second, as was pointed out by Mathis (1986), the timescale for establishing the ionization equilibrium is so long that supernovae are likely to occur in the meantime, so shocks must happen. Clearly, further modelling 
in this area would be useful. Also the role of runaway $\mathrm{O}$ stars may be important and deserves further study.

\section{MORPHOLOGY OF THE INTERSTELLAR MEDIUM AND GALAXY TYPE}

An important issue, both for the explanation of the thick $\mathrm{H} \alpha$ disks and the general disk-halo interaction, is the question of which galaxies are likely to contain star forming regions that are large enough to produce breakout of the disk and manage to vent material into the halo. One crucial parameter is the number of HII regions in a given galaxy that produces a large enough number of supernovae so that this may occur. An HII region with an observed $\mathrm{H} \alpha$ luminosity of $10^{38} \mathrm{ergs} / \mathrm{s}\left(L_{38}\right)$, corresponds to the equivalent of about 4 OV stars (Kennicutt, 1988), assuming $1 \mathrm{mag}$ of extinction in $\mathrm{H} \alpha$. Depending on the lower mass cutoff and Initial Mass Function, this translates to about 40-60 supernovae, roughly the minimum amount required to produce superbubbles, according to theoretical models (see Norman, these proceedings). Blowout will require significantly higher numbers, especially since the thickness of the disk of interstellar material is much larger than assumed in most model calculations, which in general do not include a thick $\mathrm{H} \alpha$ disk.

From the study of the HII region luminosity functions by Kennicutt et al. (1989), it is possible to estimate the number of HII regions in different galaxies, brighter than this characteristic luminosity ( $c f$. Heiles, 1990). This number does not directly translate into the expected number of superbubbles or chimneys, because the timescales involved in creating the bubble are somewhat longer than the HII region phase, but the numbers will not be different by an order of magnitude. The important result of the study by Kennicutt et al. is the strong dependence of the luminosity function, in particular the number of bright HII regions, on galaxy type. Thus, Sa and Sab galaxies do not have HII regions brighter than $5 L_{38}$ so they may never be able to produce blowout. Relatively quiescent galaxies such as M31 and M81 have of order $50 \mathrm{HII}$ regions brighter than $L_{38}$, and again few blowout regions are expected. Luminous Sc galaxies, such as M51 and M101 have some 300-500 regions brighter than $L_{38}$ and blowout is certainly likely to occur here, although not in hundreds of cases. The main point is that although blowout and chimney phenomenona are likely to occur in galaxies, most of the mass transfer between disk and halo may occur in relatively few, large events rather than in hundreds of small regions. Further observational studies can certainly address this issue. For example, it is striking that most of the $\mathrm{H} \alpha$ shells in the highly inclined galaxy M31 are quite circular in shape, suggesting they are still intrinsically spherical, hence have not reached blowout. The HI holes in M31, cataloged by Brinks and Bajaja (1986), do show some departures from sphericity, but holes larger than $300 \mathrm{pc}$ show only moderate flattening, the average axial ratio being about 0.75 . 


\section{FUTURE DEVELOPMENTS}

Further observations will greatly improve our understanding of the distribution of the WIM in galaxies of various types. Observations in other emission lines and correlation of the distribution of the WIM with the young stellar population will allow us to test the photo ionization model. More work needs to be done on trying to derive the actual morphology of the WIM; is it mainly filamentary or sheetlike? How often does blowout occur, and can we find clear regions where it is happening? What is the exact role of the WIM in the chimney or fountain models? One parameter that will be hard to get a handle on, unfortunately, is the electron density in the WIM, since the canonical methods using forbidden line ratios do not discriminate at the low electron densities in the WIM. Yet, that parameter is crucial for deriving filling factors. The connection between the WIM and the hotter ionized gas in the halo, apparent in UV absorption lines (Savage, these proceedings), needs to be studied. Further theoretical work on the modelling of the ionization would be very useful to see if we really understand the ionization mechanism. Finally, current estimates for the filling factor of the WIM seem to indicate that it fills a substantial fraction of the volume in interstellar space; models for the ISM are required that can account for this.

\section{ACKNOWLEDGEMENTS}

My work in this area has greatly benefitted from discussions with R. Braun, R.J. Dettmar, J. Hester, R.C. Kennicutt, and C. Norman. I received support from NASA grant NAS5-28086. I am grateful to the IAU for partial financial support to come to this meeting.

\section{REFERENCES}

Beuermann, K., Kanbach, G., Berkhuijsen, E.M. (1985) Astr. Ap. 153, 17

Blair, W.P., Kirshner, R.P., Chevalier, R.A. (1981) Ap.J. 247, 879

Brand, P.W.J.L., Mathis, J.S. (1978) Ap.J. 223, 161

Brinks, E., Bajaja, E. (1986) Astr. Ap. 169, 14

Courtès, G., Petit, H., Sivan, J.-P., Dodonov, S., Petit, M. (1988) Astr. Ap. 174, 28

Davies, R.D., Elliott, K.H., Meaburn, J. (1976) Mem. R. Astr. Soc. 81, 89

Dettmar, R.-J. (1990) Astr. Ap. (Lett.) 232, L15

Freedman, W.L. (1984) Ph.D. thesis, University of Toronto

Heiles, C. (1990) Ap.J. 354, 483

Hester, J.J., Kulkarni, S.R. (1990) to be published in The Interstellar Medium in External Galaxies, Poster Proc., ed. D. Hollenbach, NASA, in press

Hodge, P.W., Kennicutt, R.C. (1983) A.J. 88, 296

Hummel, E., Sancisi, R., Ekers, R.D. (1984) Astr. Ap. 133, 1

Hunter, D.A. (1982) Ap. J. 260, 81

Hunter, D.A., Gallagher, J.S. (1990) Ap.J., in press

Kennicutt, R.C. (1983) Ap.J. 272, 54

Kennicutt, R.C. (1988) Ap.J. 334, 144 
Kennicutt, R.C. (1989) Ap.J. 344, 685

Kennicutt, R.C., Edgar, B.K, Hodge, P.W. (1989) Ap. J. 337, 761

Kennicutt, R.C., Hodge, P.W. (1986) Ap. J. 306, 130

Lasker, B.M. (1977) Ap. J. 212, 390

Lasker, B.M. (1979) Pub. A.S.P. 91, 153

Mathis, J.S. (1986) Ap.J. 301, 423

Meaburn, J. (1980) M.N.R.A.S. 192, 365

Monnet, G. (1971) Astr. Ap. 12, 379

Norman, C.A., Ikeuchi, S. (1989) Ap.J. 345, 372

Pellet, A., Astier, N., Viale, A., Courtès, G., Maucherat, A., Monnet, G., Simien, F. (1978) Astr. Ap. Suppl. 31, 439

Rand, R.J., Kulkarni, S.R., Hester, J.J. (1990) Ap.J. (Lett.) 352, L1

Reynolds, R.J. (1985) Ap.J. 294, 256

Reynolds, R.J. (1987) Ap.J. 323, 118

Reynolds, R.J. (1988) Ap.J. 333, 341

Rice, W.A., Lonsdale, C.J., Soifer, B.T., Neugebauer, G., Koplan, E.L., Lloyd, L.A., de Jong, T., Habing, H.J. (1988) Ap.J.Suppl. 68, 91

Sabbadin, F., Bianchi, A. (1979) Pub. A.S.P. 91, 281

van der Kruit, P.C. (1984) Astr. Ap. 140, 470

Walterbos, R.A.M. (1988) in Galactic and Extragalactic Star Formation, eds. R.E. Pudritz and M. Fich, NAT0 ASI Series, Kluwer, 361

Walterbos, R.A.M., Braun, R. (1990a) to be published in The Interstellar Medium in External Galaxies, Poster Proc., ed. D. Hollenbach, NASA, in press

Walterbos, R.A.M., Braun, R. (1990b), in prep. 